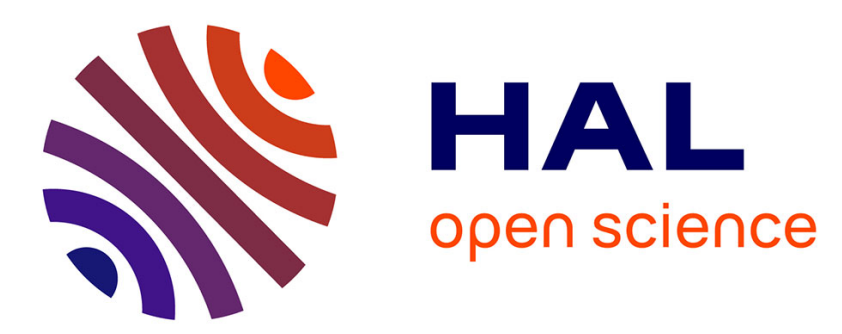

\title{
Newton's identities for minimum codewords of a family of alternant codes
}

\author{
Daniel Augot
}

\section{To cite this version:}

Daniel Augot. Newton's identities for minimum codewords of a family of alternant codes. 1995 IEEE International Symposium on Information Theory, Sep 1995, Whistler, Canada. pp.349, 10.1109/ISIT.1995.550336 . inria-00509424

\section{HAL Id: inria-00509424 https://hal.inria.fr/inria-00509424}

Submitted on 12 Aug 2010

HAL is a multi-disciplinary open access archive for the deposit and dissemination of scientific research documents, whether they are published or not. The documents may come from teaching and research institutions in France or abroad, or from public or private research centers.
L'archive ouverte pluridisciplinaire HAL, est destinée au dépôt et à la diffusion de documents scientifiques de niveau recherche, publiés ou non, émanant des établissements d'enseignement et de recherche français ou étrangers, des laboratoires publics ou privés. 


\title{
Newton's identities for minimum codewords of a family of alternant codes (extended abstract for submission)
}

\author{
Daniel Augot*
}

\begin{abstract}
We consider systems of algebraic equations which, in some way, define the minimum weight codewords of alternant codes. Results are presented which are natural generalization of the case of cyclic code [1]. Particular attention is devoted to classical Goppa codes, and a short example from [5] is presented. We use the tool of Gröbner bases for counting and describing solutions of algebraic systems [2].
\end{abstract}

\section{Fourier Transform of words on length $n$}

\subsection{Basic Properties}

Let $G F(q)$ be a finite field, let $n$ be an integer prime to $q$. We fix $\alpha$ a primitive $n$-th root of unity, which belongs to $G F\left(q^{\prime}\right)$, an extension of $G F(q)$. The word $c=\left(c_{0}, \ldots, c_{n-1}\right)$ is identified with the polynomial $c_{0}+c_{1} X+\ldots+$ $c_{n-1} X^{n-1} \bmod X^{n}-1$. The Fourier Transform of $c \in G F\left(q^{\prime}\right)^{n}$, denoted $\phi(c)$, is $A=\left(A_{0}, A_{1}, \ldots, A_{n-1}\right), A_{i}=a\left(\alpha^{i}\right), i=0 \ldots n-1$. We denote by $\odot$ the component-wise product in $G F\left(q^{\prime}\right)^{n}$. Some properties of the Fourier transform are summarized:

Theorem 1 Let $a=\left(a_{0}, a_{1}, \ldots, a_{n-1}\right) \in G F\left(q^{\prime}\right)^{n}$ and let $A=\phi(a)$ be the Fourier transform of $a$. Then $a_{i}=\frac{1}{n} A\left(\alpha^{-i}\right), i=0 \ldots n-1$. The word $a$ belongs to $G F(q)^{n}$ if and only if $A_{i q \bmod n}=A_{i}^{q}, i=0 \ldots n$ ("conjugacy constraints"[3]).

\footnotetext{
*Institut National de Recherche en Informatique et Automatique (INRIA) Domaine de Voluceau, Rocquencourt, BP 105, 78153 Le Chesnay Cedex FRANCE
} 
If $a, b$ are two words of $G F\left(q^{\prime}\right)^{n}$, then $\phi(a b)=\phi(a) \odot \phi(b), \phi(a \odot b)=$ $\frac{1}{n} \phi(a) \phi(b)$.

\subsection{Newton's Identities}

Definition 1 Let $c=\left(c_{0}, \ldots, c_{n-1}\right) \in G F\left(q^{\prime}\right)^{n}$. The locators of $c$ are $\left\{X_{1}, \ldots, X_{w}\right\}=\left\{\alpha^{i_{1}}, \ldots, \alpha^{i_{w}}\right\}$, where $i_{1}, \ldots, i_{w}$ are the indices of non zero coordinates of $c$. The elementary symmetric functions of $c$, denoted by $\sigma_{1}, \ldots, \sigma_{w}$, are $\sigma_{i}=(-1)^{i} \sum_{1 \leq j_{1}<\ldots<j_{i} \leq w} X_{j_{1}} \cdots X_{j_{i}}, i=1 \ldots w$.

The elementary symmetric functions of $c$ and the Fourier transform of $c$ are related by the (generalized) Newton's identities.

Theorem 2 [5] Let $c \in G F\left(q^{\prime}\right)^{n}$ be a word of weight $w, A=\left(A_{1}, \ldots, A_{n}\right)$ be the Fourier transform of $c$ and $\sigma_{1}, \ldots, \sigma_{w}$ the elementary symmetric functions of $c$. Then $\forall i \geq 0, \quad A_{i+w}+\sigma_{1} A_{i+w-1}+\ldots+\sigma_{w} A_{i}=0$.

A converse property is easy to prove:

Theorem 3 Let $c \in G F\left(q^{\prime}\right)^{n}$ and suppose that there exists $\gamma_{1}, \ldots, \gamma_{w}$ such that $\forall i \geq 0, \quad A_{i+w}+\gamma_{1} A_{i+w-1}+\ldots+\gamma_{w} A_{i}=0$. Then the weight of $c$ is lower or equal to $w$.

\subsection{Spectral Definition of a Code}

We consider codes $C$ of length $n$ over $G F\left(q^{\prime}\right)$. The Reed-Solomon code, denoted $R S_{k}$ is the code of length $n=q^{\prime}-1$ over $G F\left(q^{\prime}\right)$, whose codewords are $\left(F\left(\alpha^{0}\right), \ldots, F\left(\alpha^{n-1}\right)\right)$, for all polynomials $F \in G F\left(q^{\prime}\right)[X], \operatorname{deg} F<k$. A parity check matrix for $R S_{k}$ is

$$
\left[\begin{array}{ccc}
1 & \ldots & 1 \\
\alpha^{0} & \ldots & \alpha^{n-1} \\
\vdots & & \vdots \\
\left(\alpha^{0}\right)^{n-k-1} & \ldots & \left(\alpha^{n-1}\right)^{n-k-1}
\end{array}\right],
$$

since the dual of $R S_{k}$ is $R S_{n-k}$ [5].

Definition 2 Let $C$ be a code in $G F\left(q^{\prime}\right)^{n}\left(\right.$ or $\left.G F(q)^{n}\right)$. If there exists l polynomials in $n$ variables $P_{1}, \ldots, P_{l}$, such that, for all $c \in G F\left(q^{\prime}\right)^{n}\left(\operatorname{or} G F(q)^{n}\right)$, $c$ belongs to $C$ if and only if $P\left(A_{0}, \ldots, A_{n-1}\right)=\ldots=P_{l}\left(A_{0}, \ldots, A_{n-1}\right)=0$, where $A=\phi(c)$, then the code has a spectral definition. The polynomials $P_{1}, \ldots, P_{l}$ are the code spectral equations. 
Note that the code may not be linear. If the polynomials $P_{1}, \ldots, P_{l}$ are linear, the code is linear. As an example, the code spectral equations of $R S_{k}$ are $A_{0}=A_{1}=\cdots=A_{n-k-1}=0$.

Our main theorem is the following.

Theorem 4 Let $C$ be a code defined by the spectral equations $P_{1}, \ldots, P_{l}$. Let $S_{C}(w)$ be the following system of equations:

$$
\begin{gathered}
P_{1}\left(A_{0}, \ldots, A_{n-1}\right)=\cdots=P_{l}\left(A_{0}, \ldots, A_{n-1}\right)=0 \\
A_{i+w}+\sigma_{1} A_{i+w-1}+\ldots+\sigma_{w} A_{i}=0, \quad i=0 . . n-1
\end{gathered}
$$

with indeterminates $\sigma_{1}, \ldots, \sigma_{w}, A_{0}, \ldots, A_{n-1}$. Let $A=\left(A_{0}, \ldots, A_{n-1}\right)$ be a solution to $S_{C}(w)$ (i.e. there exists $\sigma_{1}, \ldots, \sigma_{w}$ such that $\left(\sigma_{1}, \ldots, \sigma_{w}, A\right)$ is a solution), then $A$ is the Fourier transform of a codeword of weight $\leq w$.

Proof: The equations $P_{1}\left(A_{0}, \ldots, A_{n-1}\right)=\cdots=P_{l}\left(A_{0}, \ldots, A_{n-1}\right)=0 \mathrm{im}$ plies that $A$ is the Fourier transform of some codeword. Theorem 3 concludes the proof.

In the particular case where $w$ is the minimum weight of $C$, we get all the Fourier transforms of minimum weight codewords.

\section{Spectral Equations for some Alternant Codes}

Definition 3 Let $\underline{\alpha}=\left(\alpha_{0}, \ldots, \alpha_{n-1}\right) \in G F\left(q^{\prime}\right)^{n}$ be distinct elements in $G F\left(q^{\prime}\right)$, and let $\underline{v}=\left(v_{0}, \ldots, v_{n-1}\right)$ be nonzero elements in $G F\left(q^{\prime}\right)$. The generalized Reed Solomon code, denoted $G R S_{k}(\underline{\alpha}, \underline{v})$, is the code whose codewords are $\left(v_{0} F\left(\alpha_{0}\right), \ldots, v_{n-1} F\left(\alpha_{n-1}\right)\right)$, for all $F \in G F\left(q^{\prime}\right)[X], \operatorname{deg} F<k$.

The alternant code $\mathcal{A}_{k}(\underline{\alpha}, \underline{v})$ is the $G F(q)$-subfield sub-code of $G R S_{k}(\underline{\alpha}, \underline{v})$.

We consider a partial class of alternant codes, the alternant codes $\Gamma(L, G)$ where $L=\left\{1, \alpha, \ldots, \alpha^{n-1}\right\}$, the set of all $n$-th roots of unity. We denote these codes $\Gamma(\alpha, \underline{v})$.

From the spectral code equations of $R S_{k}$, we derive spectral code equations for $G R S_{k}$ and $\mathcal{A}_{k}(\alpha, \underline{v})$. Let $c$ be a codeword of $G R S_{k}(\alpha, \underline{v})$, let $A=$ $\left(A_{0}, \ldots, A_{n-1}\right)=\phi(c), H=\left(H_{0}, \ldots, H_{n-1}\right)=\phi(h), A^{\prime}=\left(A_{0}^{\prime}, \ldots, A_{n-1}^{\prime}\right)=$ $\phi(h \odot c)$. Since $h \odot c$ belongs to the $R S_{k}$ code, we have $A_{0}^{\prime}=A_{1}^{\prime}=\ldots=$ $A_{n-k-1}^{\prime}=0$. Thus, using theorem 1 , the Fourier transform of the codewords of $G R S_{k}(\alpha, \underline{v})$ satisfy $\sum_{i+j=t} \bmod n A_{i} H_{j}=0, t=0 \ldots n-k-1$. In the case of the alternant code $\mathcal{A}(\alpha, \underline{v})$, the Fourier transforms of the codewords satisfy the "conjugacy constraints". 


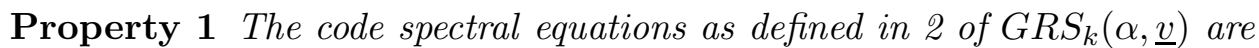
$\sum_{i+j=t \bmod n} A_{i} H_{j}=0, t=0 \ldots n-k-1$. The code spectral equations of $\mathcal{A}_{k}(\alpha, \underline{v})$ are

$$
\left\{\begin{array}{c}
\sum_{i+j=t \bmod n} A_{i} H_{j}=0, t=0 \ldots n-k-1 \\
A_{i q \bmod n}=A_{i}^{q}, i=0 \ldots n-1
\end{array}\right.
$$

where $H$ is the Fourier transform of $h$ defining the dual of the $G R S_{k}(\underline{v})$.

We consider a particular class of the classical Goppa codes: the codes $\Gamma(L, G)$, where $L=\left\{\alpha^{i}, i=0 \ldots n-1\right\}$. It is known ([5, p. 339-340]) that $\Gamma(L, G)$ is an alternant code which is the subfield sub-code of the dual of $G R S_{r}(\alpha, h)$ with $h=\left(G\left(\alpha^{0}\right)^{-1}, \ldots, G\left(\alpha^{n-1}\right)^{-1}\right)$. We compute $H=\phi(h)$, the Fourier transform of $h$. The polynomial $H$ is in fact the inverse modulo $Z^{n}-1$ of $\tilde{G}(Z)=g_{0}+\sum_{i=1}^{n-1} g_{n-i} Z^{i}$, where $G=\sum_{i=0}^{n-1} g_{i} Z^{i}$. Then the code spectral equations can be constructed.

The next section shows with an example how to deal with Goppa codes with support $L=\{0\} \cup\left\{\alpha^{i}, i=0 \ldots n-1\right\}$.

\section{An example of a Goppa Code}

We study the Goppa code of length 32 , and with defining polynomial $g(x)=$ $x^{3}+x+1$. We index codewords $c$ in the following way: $c=\left(c_{\infty}, c_{0}, \ldots, c_{30}\right)$, where the defining set of the Goppa code is $L=\left\{0,1, \alpha, \ldots, \alpha^{30}\right\}$.

First we consider the sub-code $C_{31}$ of $C$ which is the shortened code with respect to the coordinate $c_{\infty}$. This code is also a Goppa code with support $L_{31}=\left\{1, \alpha, \ldots, \alpha^{30}\right\}$ and defining polynomial $g(X)$. Thus writing the system $S_{C_{31}}(7)$, we get equations for codewords such that $c_{\infty}=0$. Computing a Gröbner basis of the system, we get 105 solutions.

Next, we want to study minimum weight codewords such $c_{\infty} \neq 0$. The parity check matrix for $C$ is

$$
G=\left[\begin{array}{cccc}
1 & g\left(\alpha^{0}\right)^{-1} & \ldots & g\left(\alpha^{30}\right)^{-1} \\
0 & \alpha^{0} g\left(\alpha^{0}\right)^{-1} & \ldots & \alpha^{30} g\left(\alpha^{30}\right)^{-1} \\
0 & \left(\alpha^{0}\right)^{2} g\left(\alpha^{0}\right)^{-1} & \ldots & \left(\alpha^{30}\right)^{2} g\left(\alpha^{30}\right)^{-1}
\end{array}\right]
$$

We search for words $c_{0}, \ldots, c_{30}$ of weight 6 , of length 31 such that $G^{\prime} c^{t}=$ $(1,0, \ldots, 0)^{t}$. where $G^{\prime}$ is the parity check matrix for $C_{31}$. Thus the spectral 
equations for these codewords are:

$$
\left\{\begin{array}{c}
\sum_{i+j=0} \bmod 31 A_{i} H_{j}=1 \\
\sum_{i+j=t \bmod 31} A_{i} H_{j}=0, t=1,2 \\
A_{2 i \bmod 31}=A_{i}^{2}, i=0 \ldots 30
\end{array}\right.
$$

These equations, plus the Newton's identities for the weight 6 , gives equations for codewords of $C$ of weight 7 whose support is not included in $[0,30]$. The Gröbner basis gives 23 solutions, thus 128 codewords of weight 7 for the whole code $C$, as in the table of [5, p344].

We point out that these Gröbner basis computations take a few minutes on a Sparc workstation.

\section{Conclusion}

Results from [1] are generalized, and applied to alternant codes whose support is the set of $n$-th roots of unity. A transform approach as in [4] could be used to generalize these results to Goppa codes with arbitrary support.

\section{References}

[1] D. Augot. Algebraic characterization of minimum weight codewords of cyclic codes. In Proceedings IEEE, ISIT'94, Trondheim, Norway, June 1994.

[2] T. Becker and V. Weispfenning. Groebner Bases, a Computationnal Approach to Commutative Algebra. Springer-Verlag, 1993.

[3] Richard E. Blahut. Theory and Practice of Error Control Codes. Addison-Wesley, 1983.

[4] Mansour Loeloeian and Jean Conan. A transform approach to Goppa codes. IEEE Transaction on Information Theory, 33(1):105-115, January 1987.

[5] F.J. Mac Williams and N.J.A. Sloane. The Theory of Error Correcting Codes. North-Holland, 1986. 